\title{
Perturbation Analysis of Rivlin-Ericksen Fluid on Heat Transfer in the Presence of Heat Absorption
}

\author{
Y. Sudarshan Reddy ${ }^{1}$, K. S. Balamurugan ${ }^{2}$ and G. Dharmaiah ${ }^{3^{*}}$ \\ ${ }^{1}$ Department of Mathematics, Sri Rajeswari College of Education, Chinnachowk, Kadapa, Andhra Pradesh, India \\ ${ }^{2}$ Department of Mathematics, RVR \& JC College of Engineering, Chowdavaram, Guntur, Andhra Pradesh, India \\ ${ }^{3}$ Department of Mathematics, Narasaraopeta Engineering College, Yellamanda, Narasaraopet, Andhra Pradesh, India \\ *Corresponding Author \\ E- Mail: dharma.g2007@gmail.com
}

\begin{abstract}
The problem of visco-elastic Rivlin-Ericksen fluid flow past a semi- infinite vertical plate embedded in a porous medium with variable temperature and suction in the presence of a uniform transverse magnetic field and thermal buoyancy effect is considered. The plate is assumed to move with a constant velocity in the direction of fluid flow while the free stream velocity is assumed to follow the exponentially increasing small perturbation law. Time-dependent wall suction is assumed to occur at the permeable surface. The dimensionless governing equations for this investigation are solved analytically using two-term harmonic and nonharmonic functions. Numerical evaluation of the analytical results is performed and some graphical results such as viscoelastic parameter $\mathbf{R m}$, heat absorption parameter $\mathbf{Q}$, Grashof number Gr, Prandtl number Pr, time t, suction velocity parameter A, moving velocity parameter $U p$ and an exponential parameter $\varepsilon$, for the velocity and temperature profiles within the boundary layer are presented. Skin-friction coefficient, Nusselt numbers are also discussed with the help of the tables.
\end{abstract}

Keywords: Variable Temperature, Vertical Plate, Suction, Heat Absorption, Rivlin-Ericksen Flow

\section{INTRODUCTION}

A few modern applications include the progression of nonNewtonian liquids, and in this manner the stream conduct of such liquids finds an incredible pertinence. Moltenmetal's, plastic, pulps, emulsions; slurries and crude materials in liquid state are a few guides to make reference to. NonNewtonian stream likewise finds functional applications in bio-designing, wherein blood flows brutal/creature vein is clarified by a fitting Visco-versatile liquid model of little flexibility. The investigation of a visco-versatile pulsatile stream helps in understanding the component of dialysis of blood through a counterfeit kidney. A significant class of two dimensional time ward stream issue managing the reaction of limit layer to outside precarious changes of the free stream speed about a mean worth pulled in the consideration of numerous specialists. Other than that convective course through permeable medium has an application in geothermal vitality recuperation, warm vitality stockpiling, oil extraction, and move through separating gadgets. Presently, magnetohydrodynamics is particularly pulling in the consideration of the numerous creators because of its applications in geophysics and building. MHD stream with warmth and mass exchange has been a subject of enthusiasm of numerous specialists in light of its different applications in science and innovation. Such wonders are seen in lightness initiated movements in the climate, in water bodies, semi strong bodies, for example, earth, and so on. The progression of viscous incompressible fluid past an indiscreetly started infinite horizontal plate in its own plane was first examined by Stokes [1]. Convective heat transfer Non-Newtonian MHD flow permeable plate inspected by the authors [2-5]. Magnificent assessments have the made on visco-elastic impact by the author [6].

An examination of viscous dissipation impacts on free convection heat and mass transfer of MHD non-Newtonian fluid flow through a permeable medium given by Nabil et al.,[7].Unsteady flow of a non-Newtonian fluid over a rotating disk with heat transfer examined by Attia [8]. MHD Rivlin-Ericksen flow through porous medium in slip stream system examined by Ramana Murthy et al., [9]. Unsteady MHD free convective heat and mass transfer flow vertical permeable plate discussed by Rao et al., [10]. Unsteady MHD free convection flow of a Kuvshinski fluid past a vertical permeable plate assumed by Reddy et al., [11]. Unsteady MHD free convection boundary layer flow of radiation on Kuvshinski fluid through porous medium was reported by Vidyasagar et al., [12]. Propelled by these examinations referenced over, the motivation behind the present work is extended the investigation of Kim [13], by considering a notable non-Newtonian fluid namely RivlinEricksen fluid for the instance of a semi-infinite moving porous plate in a permeable medium. We likewise consider the free stream to comprise of a mean velocity and temperature over which are superimposed an exponentially shifting with time. The obtained outcomes here are in great concurrence with the results of Kim without non-Newtonian fluid. The problem of visco-elastic Rivlin-Ericksen fluid past a semi- infinite vertical plate embedded in a permeable medium with variable temperature and suction in the presence of a uniform transverse magnetic field and thermal buoyancy effect is assumed. The plate is considered to move with a steady velocity in the direction of fluid flow while the free stream velocity is expected to follow the exponentially expanding small perturbation law. Timedependent wall suction is accepted to happen at the 
penetrable surface. The dimensionless governing conditions for this examination are unraveled diagnostically utilizingtwo-term harmonic and non-harmonic functions. Numerical assessment of the scientific results is performed and some graphical outcomes, such as visco-elastic parameter Rm, heat absorption parameter Q, Grashof number Gr, Prandtl number Pr, time t, suction velocity parameter A, moving velocity parameter $\mathrm{Up}$ and an exponential parameter $\varepsilon$, for the velocity and temperature profiles within the boundary layer are exhibited. Skinfriction coefficient and Nusselt numbers are additionally examined with the assistance of the tables.

\section{FORMULATION OF THE PROBLEM}

We consider transient 2-dimensional flow of a laminar, incompressible, viscous, electrically conducting and heat absorbing Rivlin-Ericksen fluid past a semi-infinite permeable vertical moving plate.

$$
\frac{\partial \tilde{u}}{\partial \tilde{t}}+\tilde{v} \frac{\partial \tilde{u}}{\partial \tilde{y}}=-\frac{1}{\rho} \frac{\partial \tilde{p}}{\partial \tilde{x}}-k_{v}\left[\frac{\partial^{3} \tilde{u}}{\partial \tilde{t} \partial \tilde{y}^{2}}+\tilde{v} \frac{\partial^{3} \tilde{u}}{\partial \tilde{y}^{3}}\right]-\frac{\sigma B_{0}^{2}}{\rho} \tilde{u}-v \frac{\tilde{u}}{\tilde{k}}+g \beta_{T}\left[\tilde{T}-\tilde{T}_{\infty}\right]+v \frac{\partial^{2} \tilde{u}}{\partial \tilde{y}^{2}}
$$$$
\frac{\partial \tilde{T}}{\partial \tilde{t}}+\tilde{v} \frac{\partial \tilde{T}}{\partial \tilde{y}}=\alpha_{d} \frac{\partial^{2} \tilde{T}}{\partial \tilde{y}^{2}}-\frac{\tilde{Q}}{\rho C p}\left[\tilde{T}-\tilde{T}_{\infty}\right]
$$

The boundary layer of equation (2) gives

$$
\frac{d \tilde{U}_{\infty}}{d \tilde{t}}+\frac{v}{\tilde{k}} \tilde{U}_{\infty}+\frac{\sigma}{\rho} \tilde{U}_{\infty} B_{0}^{2}=-\frac{1}{\rho} \frac{\partial \tilde{p}}{\partial \tilde{x}}
$$

Corresponding boundary conditions are

$$
\begin{aligned}
& \tilde{u}=\tilde{u}_{p}, T=T_{w}+\varepsilon e^{\tilde{n} \tilde{t}}\left[\tilde{T}_{w}-\tilde{T}_{\infty}\right] \text { at } \tilde{y}=0 \\
& \tilde{u} \rightarrow \tilde{u}_{\infty}=U_{0}\left[1+\varepsilon e^{\tilde{n} \tilde{t}}\right], T \rightarrow T_{\infty} \text { as } \tilde{y} \rightarrow \infty
\end{aligned}
$$

The suction velocity at the plate surface of equation (1) is a function of time only so it assumes

$$
\tilde{v}=-V_{0}\left(1+\varepsilon A e^{\tilde{n} \tilde{t}}\right)
$$

Where $\tilde{x}$ - the dimensional distances along the plate, $\tilde{y}$ the dimensional distance perpendicular to the plate, $\tilde{t}$ dimensional time, $\tilde{u}$ - the component of dimensional velocity along $\tilde{x}$ direction, $\tilde{v}$ - the component of dimensional velocity along $\tilde{y}$ direction, $\rho-$ the fluid density, $v$ - the kinematic viscosity, $\mathrm{Cp}$ - the specific heat at constant pressure, $\sigma-$ the fluid electrical conductivity, $B_{0}$ magnetic induction, $\tilde{k}$ - the permeability of the porous medium, $\mathrm{T}$ - the dimensional temperature, $\mathrm{Q}_{0}-$ the dimensional heat absorption coefficient, $\alpha_{d}$-the thermal diffusivity, $\mathrm{g}$ - gravitational acceleration, $\beta_{T}-$ the coefficient of volumetric expansion, $k_{v}$ - the kinematic visco-elasticity, A - a real positive constant, $\varepsilon \& \varepsilon A-$ less than unity values, $\mathrm{V}_{0}-\mathrm{a}$ scale of suction velocity which has non-zero positive constant.
Here we considered the following:

1. There is no applied voltage that implies the absence of an electrical field.

2. The magnetic field and Reynolds number are very small.

3. The Hall effects are negligible.

4. Viscous dissipation is neglected.

5. Plate moves with a constant velocity in the direction of fluid flow.

6. The temperature at the wall as well as the suction velocity is exponentially varying with time.

The governing equations can be written in Cartesian frame of reference as:

$$
\frac{\partial \tilde{v}}{\partial \tilde{y}}=0
$$

Employed dimensionless variables are:

$$
\begin{aligned}
& u=\frac{\tilde{u}}{U_{0}}, v=\frac{\tilde{v}}{V_{0}}, \eta=\frac{V_{0} \tilde{y}}{v}, U_{\infty}=\frac{\tilde{U}_{\infty}}{U_{0}}, R_{m}=\frac{V_{0}^{2} k_{v}}{v^{2}}, k=\frac{\tilde{k} V_{0}^{2}}{v^{2}}, \\
& t=\frac{\tilde{t} V_{0}^{2}}{v}, \theta=\frac{\tilde{T}-\tilde{T}_{\infty}}{\tilde{T}_{w}-\tilde{T}_{\infty}}, n=\frac{\tilde{n} v}{V_{0}^{2}}, \operatorname{Pr}=\frac{v}{\alpha_{d}}=\frac{v \rho C_{p}}{k}, \\
& M=\frac{\sigma v B_{0}^{2}}{\rho V_{0}^{2}}, G r=\frac{v \beta_{T} g\left[\tilde{T}_{w}-\tilde{T}_{\infty}\right]}{U_{0} V_{0}^{2}}, Q=\frac{v Q_{0}}{V_{0}^{2} \rho C_{p}}
\end{aligned}
$$

Substituting using equation (8) along with (4) to (7) in equations (1) and (2), we can obtain dimensionless forms in the following manner:

$$
\begin{aligned}
& \frac{\partial u}{\partial t}-\left[1+\varepsilon A e^{n t}\right] \frac{\partial u}{\partial \eta}= \frac{d U_{\infty}}{d t}-R m\left[\frac{\partial^{3} u}{\partial t \partial \eta^{2}}-\left[1+\varepsilon A e^{n t}\right] \frac{\partial^{3} u}{\partial \eta^{3}}\right]_{(9)} \\
&+N_{1}\left[U_{\infty}-u\right]+G r \theta+\frac{\partial^{2} u}{\partial \eta^{2}} \\
& \operatorname{Pr}\left(\frac{\partial \theta}{\partial t}-\left[1+\varepsilon A e^{n t}\right] \frac{\partial \theta}{\partial \eta}\right)=\frac{\partial^{2} \theta}{\partial \eta^{2}}-\operatorname{Pr} Q \theta
\end{aligned}
$$

The corresponding dimensionless boundary conditions are

$$
\begin{aligned}
& u=U_{p}, \theta=1+\varepsilon e^{n t} \quad \text { at } \quad \eta=0 \\
& u \rightarrow U_{\infty}=U_{0}\left[1+\varepsilon e^{n t}\right] \quad \text { as } \eta \rightarrow \infty
\end{aligned}
$$

Where

$N_{1}=M+\frac{1}{k}$

Also $\mathrm{Gr}$ - the solutal Grashof number, $\mathrm{Pr}$ - Prandtl number, $\mathrm{Q}$ - dimensionless heat absorption coefficient. 


\section{SOLUTION OF THE PROBLEM}

Equations (9) \& (10) represent a set of partial differential equations that cannot be solved in closed form. But, it can be reduced to a set of ordinary differential equations in dimensionless from that can be solved analytically. This can be done by taking in the following way.

$$
\begin{gathered}
u(\eta, t)=l_{0}(\eta)+\varepsilon e^{n t} l_{1}(\eta)+\ldots \ldots \ldots . . \\
R_{m} l_{1}^{\prime \prime \prime}+\left[1-n R_{m}\right] \\
m_{0}^{\prime \prime}+\operatorname{Pr} m_{0}^{\prime}-Q \operatorname{Pr} m_{0}=0 \\
m_{1}^{\prime \prime}+\operatorname{Pr} m_{1}^{\prime}-(n+Q) \operatorname{Pr} m_{1}=-A \operatorname{Pr} m_{0}^{\prime}
\end{gathered}
$$$$
R_{m} l_{1}^{\prime \prime \prime}+\left[1-n R_{m}\right] l_{1}^{\prime \prime}+l_{1}^{\prime}-\left[N_{1}+n\right] l_{1}=-\left[n+N_{1}\right]-m_{1} G r-A R_{m} l_{0}^{\prime \prime \prime}-A l_{0}^{\prime}(16)
$$

The corresponding boundary conditions are

$$
\begin{aligned}
& l_{0}=U_{p}, l_{1}=0, m_{0}=1, m_{1}=1 \quad \text { at } \quad \eta=0 \\
& u(\eta, t)=l_{0}(\eta)+\varepsilon e^{n t} l_{1}(\eta) \\
&=\left[l_{01}+R_{m} l_{02}\right]+\varepsilon e^{n t}\left[l_{1}\right. \\
&=\left[\left(h_{4} e^{-z_{6} \eta}+h_{3} e^{-z_{1} \eta}+1\right)\right. \\
& \varepsilon e^{n t}\left[\begin{array}{l}
\left(-h_{16} e^{-z_{10} \eta}+h_{14}\right. \\
R_{m}\left(-h_{27} e^{-z_{12} \eta}+\right.
\end{array}\right. \\
& \theta(\eta, t)=m_{0}(\eta)+\varepsilon e^{n t} m_{1}(\eta) \\
&=\left(e^{-z_{1} \eta}\right)+\varepsilon e^{n t}\left(h_{2} e^{-z_{3} \eta}+h_{1} e^{-z_{1} \eta}\right)
\end{aligned}
$$$$
=\left[l_{01}+R_{m} l_{02}\right]+\varepsilon e^{n t}\left[l_{11}+R_{m} l_{12}\right]
$$$$
=\left[\left(h_{4} e^{-z_{6} \eta}+h_{3} e^{-z_{1} \eta}+1\right)+R_{m}\left(h_{7} e^{-z_{8} \eta}+h_{5} e^{-z_{6} \eta}+h_{6} e^{-z_{1} \eta}\right)\right]+
$$$$
\varepsilon e^{n t}\left[\begin{array}{l}
\left(-h_{16} e^{-z_{10} \eta}+h_{14}+h_{9} e^{-z_{3} \eta}+h_{15} e^{-z_{1} \eta}+h_{12} e^{-z_{6} \eta}\right)+ \\
R_{m}\left(-h_{27} e^{-z_{12} \eta}+h_{22} e^{-z_{6} \eta}+h_{23} e^{-z_{1} \eta}+h_{24} e^{-z_{8} \eta}+h_{25} e^{-z_{10} \eta}+h_{26} e^{-z_{3} \eta}\right)
\end{array}\right]
$$

$$
\theta(\eta, t)=m_{0}(\eta)+\varepsilon e^{n t} m_{1}(\eta)+\ldots \ldots \ldots
$$

Substituting equations (13) and (14) into equations (9) \& (10), equating like terms and ignoring higher order terms, we can get the following pairs of equations for $\left(l_{0}, m_{0}\right) \&$ $\left(l_{1}, m_{1}\right)$.

$$
R_{m} l_{0}^{\prime \prime}+l_{0}^{\prime \prime}+l_{0}^{\prime}-N_{1} l_{0}=-\left[N_{1}+m_{0} G r\right]
$$

$$
l_{0}=1, l_{1}=1, m_{0} \rightarrow 0, m_{1} \rightarrow 0 \quad \text { as } \quad \eta \rightarrow \infty
$$

Where prime denotes ordinary differentiation with respect to $\eta$. Without going into detail, the velocity and temperature distributions in the boundary layer are
The skin-friction coefficient and Nusselt number for this boundary layer flow can be defined as:

$$
\tau=\left(\frac{\partial u}{\partial \eta}\right)_{\eta=0} \text { and } N u=-\left(\frac{\partial \theta}{\partial \eta}\right)_{\eta=0}
$$

\section{RESULTS AND DISCUSSION}

The objective of the present paper is to investigate the effects visco-elastic Rivlin-Ericksen fluid flow past an semiinfinite vertical plate embedded in a porous medium with variable temperature and suction. In order to get a clear insight of the physical problem, the numerical computations have been carried out for various values of material parameters such as visco-elastic parameter Rm, heat absorption parameter Q, Grashof number Gr, Prandtl number Pr, time $t$, suction velocity parameter A, moving velocity parameter $\mathrm{Up}$ and an exponential parameter $\varepsilon$, which are of physical and engineering interest. The numerical results are displayed with the help of graphical illustrations in figures 1-13. The parameters $\operatorname{Pr}=0.71, \mathrm{n}=$ $1, \mathrm{Q}=1$ are kept fixed throughout the discussion.

Fig.1 presents the variation of the velocity distribution across the boundary layer for different values of the plate velocity Up in the direction of the fluid flow. Although we have different initial plate velocities, the velocity increases to the constant value for given material parameters. Fig. 2 shows the effect of Prandtl number (Pr) on the velocity profiles. It is observe that the velocity decreases with increasing values of Prandtl number (Pr). It is of great interest to show how the time $t$ effects on velocity and temperature. It is observed that velocity profile $\mathrm{u}$ increases strongly far field regime in Fig. 3, temperature profile $\theta$ increases strongly near and far field regime in Fig. 10. Fig.4 presents the variation of the velocity distribution across the boundary layer for different values of the plate velocity $\varepsilon$ in the direction of the fluid flow. The velocity increases as $\varepsilon$ is increasing.

Effect of heat absorption parameter on velocity is presented in fig.5. From this figure, it is noticed that velocity decreases with an increase in heat absorption parameter. Fig.6 exhibits the effects of visco-elastic parameter on fluid velocity for the flow past a cooled plate $(\mathrm{Gr}>0)$. With the amplification of visco-elastic parameter, the velocity pattern 
for cooled plate gradually decreases on velocity components. It may be concluded that the energy due elastic property of the fluid increases the velocity and then gets dissipated. Grashof number studies the behavior of free convection and it is defined as the ratio of buoyancy force to viscous force. It plays an important role in heat and mass transfer technology. In this study, the results are discussed for the flow past an externally cooled plate $(\mathrm{Gr}>0)$ and flow past an externally heated plate $(\mathrm{Gr}<0)$. Fig.7 is depicted for positive values of the buoyancy parameter $\mathrm{Gr}$ which corresponds to the cooling problem. The cooling problem is often encountered in the cooling of nuclear reactors. It is experienced from Fig. 7 that the velocity rises as increasing values of Grashof number (Gr). Fig.8 presents the variation of the temperature distribution across the boundary layer for different values of the plate temperature $\varepsilon$ in the direction of the fluid flow. The temperature increases as $\varepsilon$ is increasing. Effect of heat absorption parameter on temperature is shown in fig. 9, from which it is concluded that the temperature decreases as heat absorption parameter increases. The effects of $\mathrm{A}$ on the temperature of the fluid are depicted through graph 11 . The temperature decreases for increase in A. The effects of $\mathrm{n}$ on the temperature of the fluid are depicted through graph 12 . The temperature increases for increase in n. Fig. 13 shows the effect of Prandtl number (Pr) on the temperature profiles. It is observe that the temperature decreases with increasing values of Prandtl number (Pr).

Effects of various parameters on skin friction and rate of heat transfer are presented in tables I-II. After knowing the velocity field, it is very important from a physical point of view to know the effect of viscoelastic parameter on resistive force or viscous drag. The resistive force or viscous drag on the surface of the body due to the motion of the fluid is known as the shearing stress or skin-friction coefficient. Table I depicts that on increasing thermal Grashof number Gr, visco-elasticity parameter Rm, Radiation absorption parameter Q, time t, Prandtl number $\operatorname{Pr}$, suction velocity parameter A and Scalar constant $\varepsilon$, skin friction coefficient $\tau$ increases. From table II, it is observed that Nusselt number increases for increasing values of radiation absorption parameter $\mathrm{Q}$, time t, Prandtl number Pr and suction velocity parameter $\mathrm{A}$.

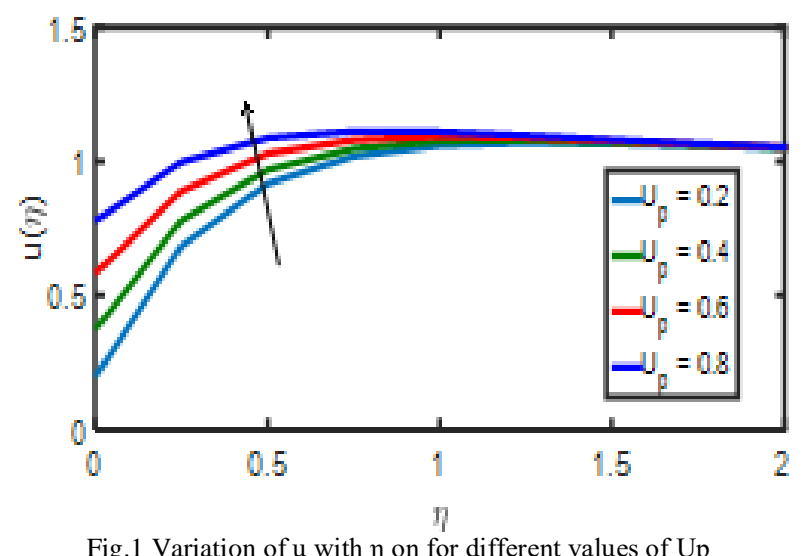

Fig.1 Variation of $u$ with $\eta$ on for different values of $U p$

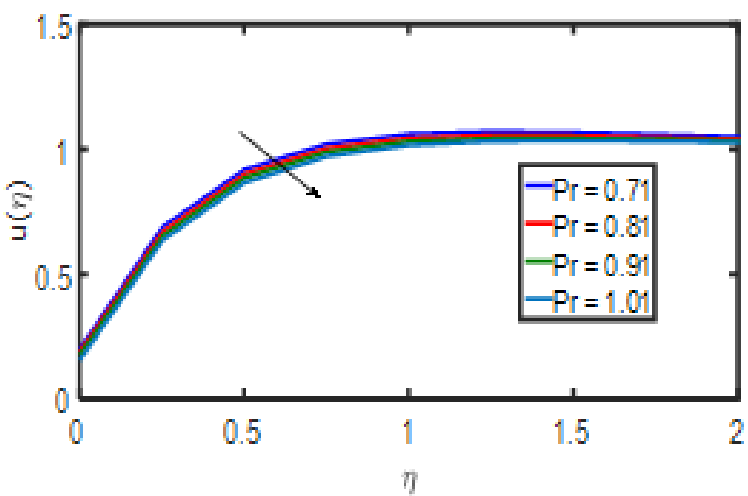

Fig.2 Variation of $u$ with $\eta$ on for different values of $\operatorname{Pr}$

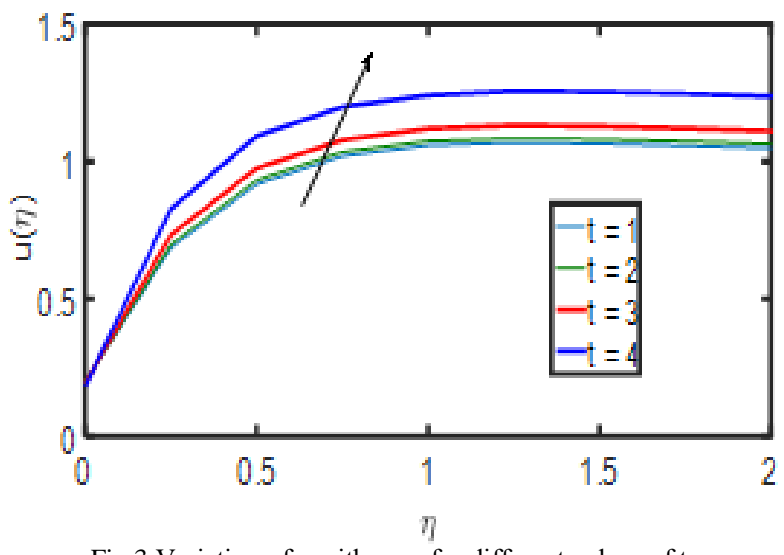

Fig.3 Variation of $u$ with $\eta$ on for different values of $t$

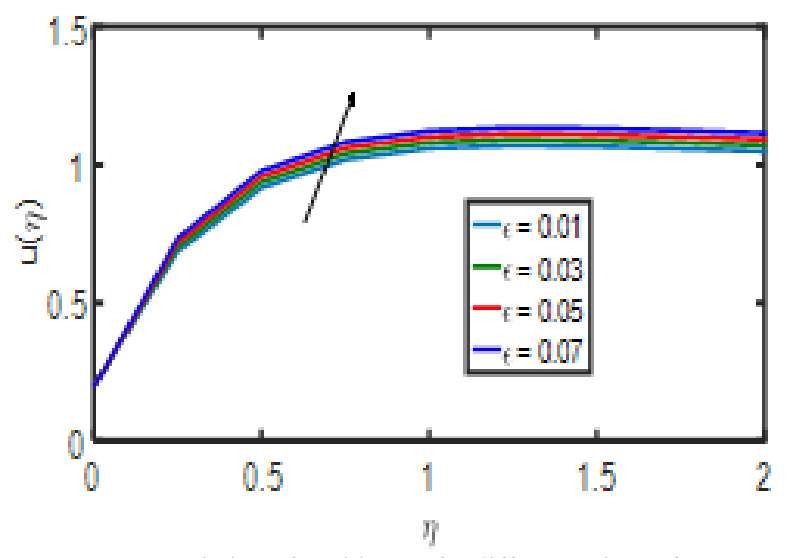

Fig. 4 Variation of $u$ with $\eta$ on for different values of $\varepsilon$

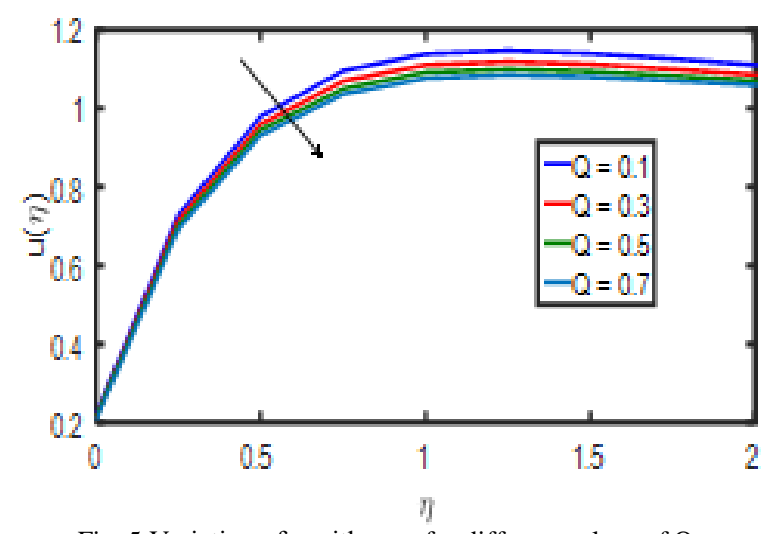

Fig. 5 Variation of $u$ with $\eta$ on for different values of $Q$ 


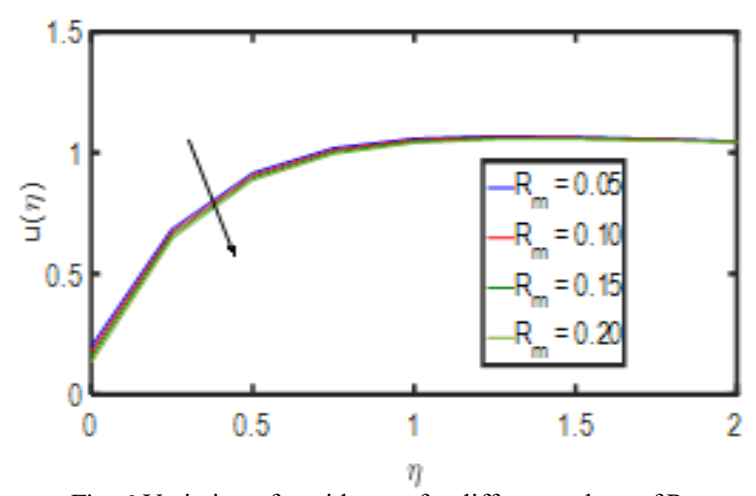

Fig. 6 Variation of $u$ with $\eta$ on for different values of $R m$

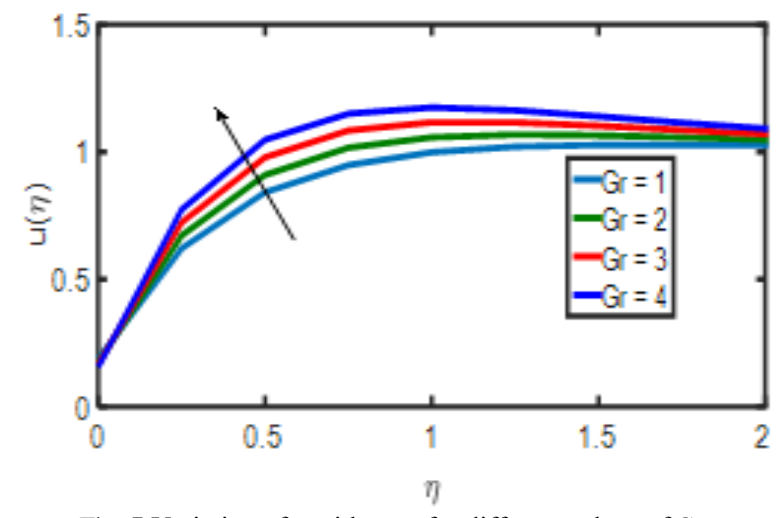

Fig. 7 Variation of $u$ with $\eta$ on for different values of $\mathrm{Gr}$

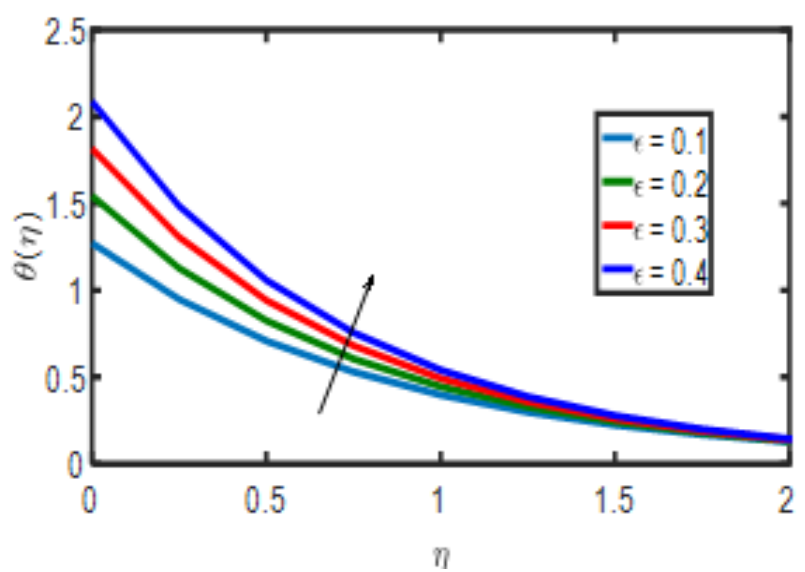

Fig. 8 Variation of $\theta$ with $\eta$ on for different values of $\varepsilon$

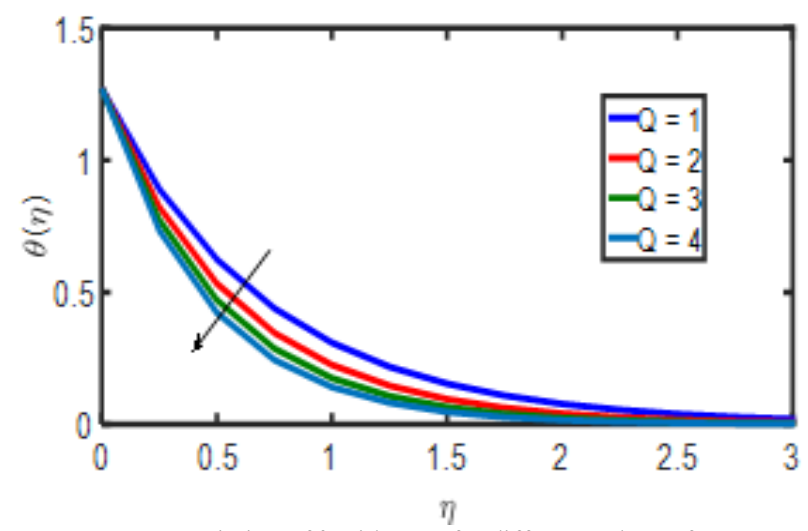

Fig. 9 Variation of $\theta$ with $\eta$ on for different values of $Q$

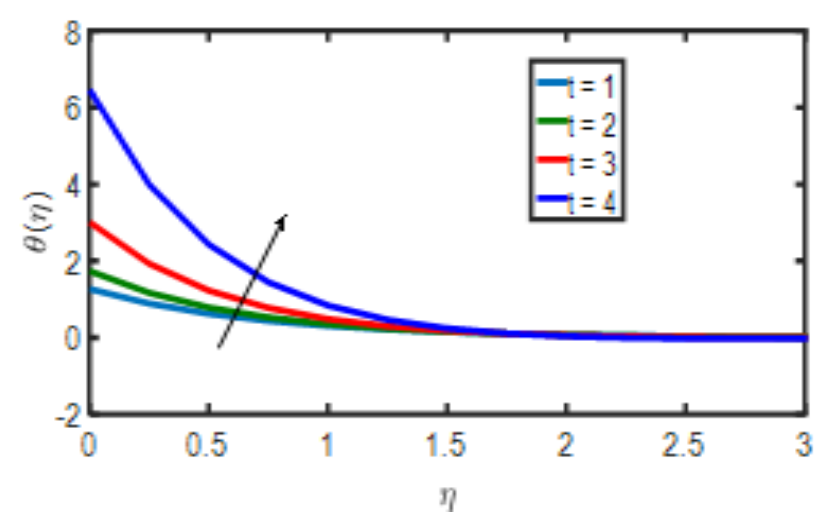

Fig. 10 Variation of $\theta$ with $\eta$ on for different values of $t$

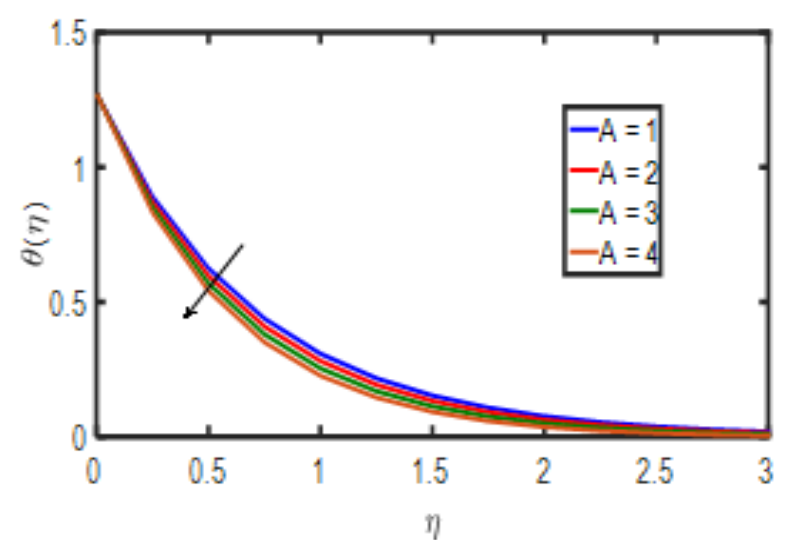

Fig. 11 Variation of $\theta$ with $\eta$ on for different values of $A$

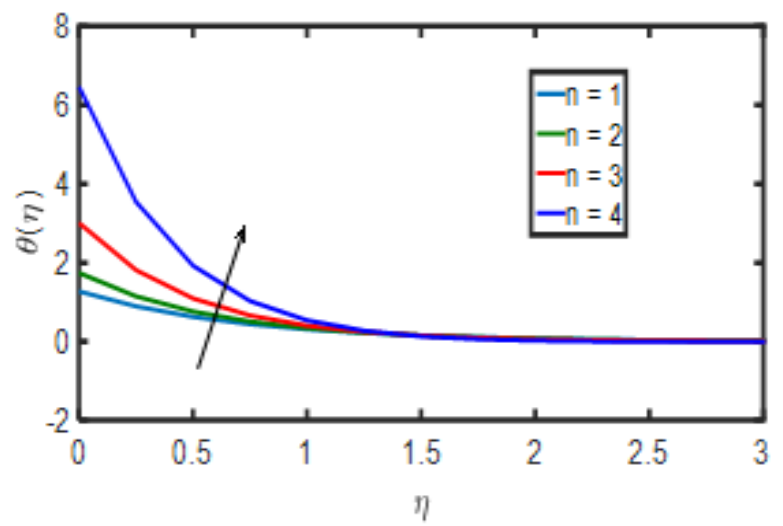

Fig. 12 Variation of $\theta$ with $\eta$ on for different values of $n$

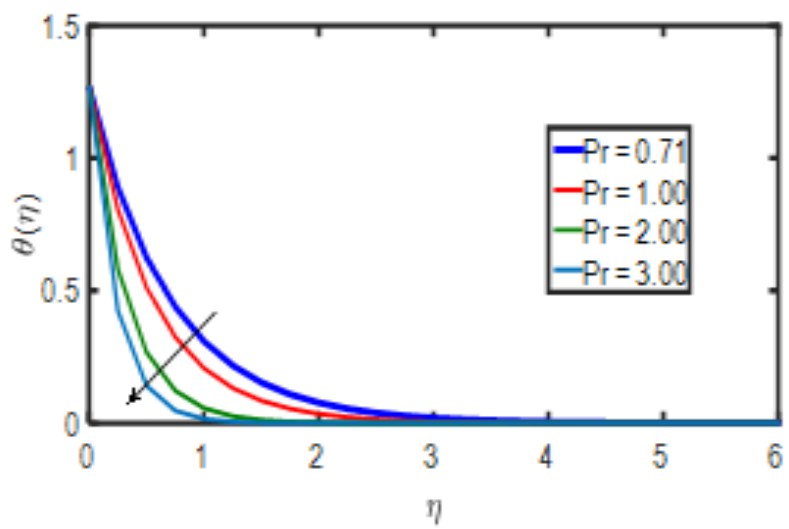

Fig. 13 Variation of $\theta$ with $\eta$ on for different values of $\operatorname{Pr}$ 
TABLE I CF FOR DIFFERENT PARAMETERS

\begin{tabular}{|c|c|c|c|c|c|c|c|}
\hline $\mathbf{G r}$ & $\mathbf{R m}$ & $\mathbf{Q}$ & $\mathbf{t}$ & $\mathbf{P r}$ & $\mathbf{A}$ & $\varepsilon$ & Cf \\
\hline $\begin{array}{l}1 \\
2 \\
3 \\
4\end{array}$ & & & & & & & $\begin{array}{l}1.1541 \\
1.4386 \\
1.7231 \\
2.0076\end{array}$ \\
\hline & $\begin{array}{l}0.01 \\
0.02 \\
0.03 \\
0.04 \\
\end{array}$ & & & & & & $\begin{array}{l}2.3082 \\
4.6163 \\
6.9245 \\
9.2326\end{array}$ \\
\hline & & $\begin{array}{l}0.1 \\
0.2 \\
0.3 \\
0.4\end{array}$ & & & & & $\begin{array}{l}1.1184 \\
1.1216 \\
1.1249 \\
1.1285\end{array}$ \\
\hline & & & $\begin{array}{l}0.2 \\
0.3 \\
0.4 \\
0.5\end{array}$ & & & & $\begin{array}{l}1.2755 \\
1.4096 \\
1.5578 \\
1.7217\end{array}$ \\
\hline & & & & $\begin{array}{l}1 \\
2 \\
3 \\
4\end{array}$ & & & $\begin{array}{l}1.2603 \\
5.4156 \\
7.8720 \\
8.2912\end{array}$ \\
\hline & & & & & $\begin{array}{l}1 \\
2 \\
3 \\
4 \\
\end{array}$ & & $\begin{array}{l}1.7435 \\
2.9224 \\
4.1012 \\
5.2801 \\
\end{array}$ \\
\hline & & & & & & $\begin{array}{l}0.02 \\
0.03 \\
0.04 \\
0.05 \\
\end{array}$ & $\begin{array}{l}2.3082 \\
3.4622 \\
4.6163 \\
5.7704 \\
\end{array}$ \\
\hline
\end{tabular}

TABLE II NU FOR DIFFERENT PARAMETERS

\begin{tabular}{|c|c|c|c|c|}
\hline $\mathbf{Q}$ & $\mathbf{t}$ & $\mathbf{A}$ & $\mathbf{P r}$ & $\mathbf{N u}$ \\
\hline 1 & & & & 1.8174 \\
2 & & & & 2.2155 \\
3 & & & & 2.5328 \\
4 & & & & 2.8050 \\
\hline & 0.1 & & & 1.4922 \\
& 0.2 & & & 1.5156 \\
& 0.3 & & & 1.5415 \\
& 0.4 & & & 1.5701 \\
\hline & & 0.1 & & 1.7152 \\
& & 0.2 & & 1.7265 \\
& & 0.3 & & 1.7379 \\
& & 0.4 & & 1.7492 \\
\hline & & & 0.71 & 1.8174 \\
& & & 1.00 & 2.3297 \\
& & & 2.00 & 3.9860 \\
& & & 3.00 & 5.5786 \\
\hline
\end{tabular}

\section{CONCLUSION}

The governing equations for combined effects of heat absorption MHD on convective Rivlin-Ericksen flow past a semi-infinite vertical porous plate with variable temperature and suction was formulated. The plate velocity was maintained at a constant value and the flow was subjected to a transverse magnetic field. Numerical results are presented to illustrate the details of the flow and heat transfer characteristics and their dependence on the material parameters. It is seen that as Up increases, velocity increases. It is seen that the velocity decreases with increasing values of Pr. It is observed that velocity profile $u$ increases strongly far field regime. It is observed the velocity increases as $\varepsilon$ is increasing. It is noticed that velocity decreases with an increase in Q. It is observed the velocity decreases as $\mathrm{Gr}$ increases. It is noticed that the temperature increases as $\varepsilon$ is increasing. It is concluded that the temperature decreases as Q. It is seen that the temperature decreases for increase in A. It is observed that the temperature decreases with increasing of Pr.

\section{REFERENCES}

[1] G. G. Stokes, "Flow of viscous incompressible fluid past an impulsively started infinite horizontal plate", Cambridge Phil. Trans., Vol. 9, pp. 8-17, 1851.

[2] K. R. Choubey and R. R. Yadav, "Magneto hydrodynamic flow of a non-newtonian fluid past a porous plate", Astrophysics and Space Science, Vol. 115, pp. 345-351, 1985.

[3] Basant Kumar Jha, "MHD free-convection and mass transfer flow of an elasto-viscous fluid", Astrophysics and Space Science, Vol. 185, pp. 129-135, 1991.

[4] N. K. Samria, R. Prasad and M. U. S. Reddy, "MHD free-convection flow of a visco-elastic fluid past an infinite vertical plate", Astrophysics and Space Science, Vol. 181, pp. 135-145, 1991.

[5] M. Hameed and Nadeem, "Unsteady mhd flow of a non-newtonian fluid on a porous plate", J.Math.Anal, Vol. 325, pp. 724-733, 2007.

[6] R. A. Damesh and B. A. Shannak, "Visco-elastic fluid flow past an infinite vertical porous plate in the presence of first order chemical reaction", App. Math. Mech., Vol. 31, pp. 955-962, 2010.

[7] T. M. Nabil, Eldabe, N. S. Sallam, Y. Mohamed and Abou-Zeid, "Numerical study of viscous dissipation effect on free convection heat and mass transfer of mhd non-newtonian fluid flow through a porous medium", Journal of Egyptian Mathematical Society, Vol. 20, pp. 139-151, 2012. 
[8] H. A. Attia, "Unsteady flow of a non-newtonian fluid above a rotating disk with heat transfer", Int. J. of Heat and Mass Transfer, Vol. 46, pp. 2695-2700, 2013.

[9] M. V. Ramanamurthy, "Magnetohydrodynamics rivlin-ericksen flow through porous medium in slip flow regime", IJSETR, Vol. 4, 2015.

[10] B. M. Rao, G. V. Reddy, M. C. Raju and S. V. K. Varma, "Unsteady mhd free convective heat and mass transfer flow past a semi-infinite vertical permeable moving plate with heat absorption, radiation, chemical reaction and soret effects", International Journal of Engineering Sciences \& Emerging Technologies, Vol. 6, pp. 241-257, 2013.

[11] S. Harinath Reddy, M. C. Raju and E. Keshava Reddy, "Unsteady mhd free convection flow of a kuvshinski fluid past a vertical porous plate in the presence of chemical reaction and heat source/sink", International Journal of Engineering Research in Africa, Vol. 14, pp. 13-27,2015.

[12] B. Vidyasagar, M. C. Raju, S. V. K. Varma and S. V. Ramana, "Unsteady mhd free convection boundary layer flow of radiation absorbing kuvshinski fluid through porous medium", Review of Advances in Physics Theories and Applications, Vol.1, pp. 48-62, 2014.

[13] Y. J. Kim, "Unsteady mhd convective heat transfer past a semiinfinite vertical porous moving plate with variable suction", Int J Eng Sci, Vol.38, pp.833-45, 2000. 